\title{
Slant Curves in 3-Dimensional Normal Almost Paracontact Metric Manifolds
}

\author{
Joanna Wełyczko
}

\begin{abstract}
The present paper is devoted to study the curvature and torsion of slant Frenet curves in 3-dimensional normal almost paracontact metric manifolds. Moreover, in this class of manifolds, properties of nonFrenet slant curves (with null tangents or null normals) are studied. We illustrate such results by some examples.
\end{abstract}

Mathematics Subject Classification (2010). 53C15, 53C50.

Keywords. Normal almost paracontact metric manifolds, Slant curves, Legendre curves, Frenet curves, null curves, curves with null normal.

\section{Introduction}

In this paper, we consider the properties of slant curves in 3-dimensional normal almost paracontact metric manifolds. Slant curves have been studied in [6-9] but a direct inspiration to this work was the paper [5], where slant curves in 3-dimensional normal almost contact manifolds are considered.

In a 3-dimensional manifold $M$ endowed with an almost contact metric structure $(\varphi, \xi, \eta, g)$ the metric $g$ is positive definite, a slant curve is definite as a unit curve $\gamma: I \rightarrow M$ for which $g(\dot{\gamma}(t), \xi)=\cos \theta(t)=$ const, where $\theta: I \rightarrow[0,2 \pi)$ is called a structural angle, therefore the notion of a slant curve generalizes the notion of cylindrical helix in Euclidean space $\mathbb{E}^{3}$ - curves known from classical differential geometry, for which the tangent vector field has a constant angle with fixed direction.

In present paper, we consider manifolds with pseudo-Riemannian metric $g$, so that our definition of a slant curve is more general and we demand $\gamma: I \rightarrow M$ be a unit or null curve and $g(\dot{\gamma}(t), \xi)=$ const.. We write about this in the first section. In view of this definition, we look at the slant curves also as a generalization of Legendre curves (see e.g. [1,2,15,21,22]). In [22], we have investigated Legendre curves in 3-dimensional normal almost paracontact metric manifolds. Some results obtained in present paper generalizes theorems for Legendre curves from [22]. Moreover, we present new properties of Legendre curves in Theorem 4.3 and Corollary 4.4. 
This work consists of five sections. In the first one we define a normal almost paracontact metric manifold as well as a slant curve in a 3-dimensional normal almost paracontact metric manifold. We also present some properties of these objects. In second, third and fourth sections, we present, respectively, definitions and curvature properties of Frenet slant curves, null slant curves and slant curves with null normal. The examples illustrating theorems are in the last section.

\section{Preliminaries}

Let $M$ be a $(2 n+1)$-dimensional differentiable manifold. Let $\varphi$ be a $(1,1)$ tensor field, $\xi$ a vector field and $\eta$ a 1-form on $M$. Then $(\varphi, \xi, \eta)$ is called an almost paracontact structure on $M$ if

(i) $\eta(\xi)=1, \quad \varphi^{2}=\mathrm{Id}-\eta \otimes \xi$,

(ii) the tensor field $\varphi$ induces an almost paracomplex structure on the distribution $\mathcal{D}=k e r \eta$, that is, the eigen distributions $\mathcal{D}^{+}, \mathcal{D}^{-}$corresponding to the eigenvalues $1,-1$ of $\varphi$, respectively, have equal dimension $n$.

$M$ is said to be almost paracontact manifold if it is endowed with an almost paracontact structure (cf. $[4,14,20,23])$.

Let $M$ be an almost paracontact manifold. $M$ will be called an almost paracontact metric manifold if it is additionally endowed with a pseudoRiemannian metric $g$ of signature $(n+1, n)$ and such that

$$
g(\varphi X, \varphi Y)=-g(X, Y)+\eta(X) \eta(Y) .
$$

For such a manifold, we additionally have $\eta(X)=g(X, \xi), \eta(\xi)=1, \varphi \xi=0$, $\eta \circ \varphi=0$. Moreover, we can define a skew-symmetric 2 -form $\Phi$ by $\Phi(X, Y)=$ $g(X, \varphi Y)$, which is called the fundamental form corresponding to the structure. Note that $\eta \wedge \Phi^{n}$ is up to a constant factor the Riemannian volume element of $M$.

On an almost paracontact manifold, one defines the $(2,1)$-tensor field $N^{(1)}$ by

$$
N^{(1)}(X, Y)=[\varphi, \varphi](X, Y)-2 d \eta(X, Y) \xi,
$$

where $[\varphi, \varphi]$ is the Nijenhuis torsion of $\varphi$ given by

$$
[\varphi, \varphi](X, Y)=\varphi^{2}[X, Y]+[\varphi X, \varphi Y]-\varphi[\varphi X, Y]-\varphi[X, \varphi Y] .
$$

If $N^{(1)}$ vanishes identically, then the almost paracontact manifold (structure) is said to be normal (cf. [20] and recent papers [4,23]). The normality condition says that the almost paracomplex structure $J$ defined on $M \times \mathbb{R}$ by

$$
J\left(X, \lambda \frac{d}{d t}\right)=\left(\varphi X+\lambda \xi, \eta(X) \frac{d}{d t}\right)
$$

is integrable (paracomplex).

In the sequel, we are interested in dimension 3. The following theorem presents conditions equivalent to the normality of 3 dimensional almost paracontact manifolds, which we will use later in the work. 
Theorem 1.1 [22]. For a 3-dimensional almost paracontact metric manifold $M$, the following three conditions are mutually equivalent

(a) $M$ is normal,

(b) there exist functions $\alpha, \beta$ on $M$ such that

$$
\left(\nabla_{X} \varphi\right) Y=\beta(g(X, Y) \xi-\eta(Y) X)+\alpha(g(\varphi X, Y) \xi-\eta(Y) \varphi X),
$$

(c) there exist functions $\alpha, \beta$ on $M$ such that

$$
\nabla_{X} \xi=\alpha(X-\eta(X) \xi)+\beta \varphi X .
$$

Here $\nabla$ is the Levi-Civita connection of $g$.

Corollary 1.2 [22]. The functions $\alpha, \beta$ appearing in (1.2) and (1.3) are given by

$$
2 \alpha=\text { Trace }\left\{X \rightarrow \nabla_{X} \xi\right\}, \quad 2 \beta=\operatorname{Trace}\left\{X \rightarrow \varphi \nabla_{X} \xi\right\} .
$$

A 3-dimensional normal almost paracontact metric manifold is said to be

- paracosymplectic if $\alpha=\beta=0$ [10],

- quasi-para-Sasakian if and only if $\alpha=0$ and $\beta \neq 0[14,22]$,

- $\beta$-para-Sasakian if and only if $\alpha=0$ and $\beta \neq 0$ and $\beta$ is constant, in particular, para-Sasakian if $\beta=-1[22,23]$,

- $\alpha$-para-Kenmotsu if $\alpha \neq 0$ and $\alpha$ is constant and $\beta=0$.

At the end of this section, let $\gamma: I \rightarrow M, I$ being an interval, be a curve on $M$ such that $g(\dot{\gamma}, \dot{\gamma})= \pm 1$ or $g(\dot{\gamma}, \dot{\gamma})=0$ and let $\nabla_{\dot{\gamma}}$ denote the covariant differentiation along $\gamma$.

We say that the curve $\gamma$ is a slant curve in a 3-dimensional normal almost paracontact metric manifold, if

$$
g(\dot{\gamma}, \xi)=\eta(\dot{\gamma})=c \text { and } c \text { is constant. }
$$

In the particular case, $c=0$ the curve $\gamma$ is called Legendre curve [22].

Remark 1.3. In a normal almost contact metric manifold with Riemannian metric $g$, the value of $g(\dot{\gamma}, \xi)$ satisfies $-1 \leqslant g(\dot{\gamma}, \xi) \leqslant 1$, so that we can define the structural angle of $\gamma$ i.e the function $\theta: I \rightarrow[0,2 \pi)$ given by

$$
\cos \theta(t)=g(\dot{\gamma}(t), \xi)=\eta(\dot{\gamma}(t)) .
$$

Then, the curve $\gamma$ is said to be a slant curve, (or $\theta$-slant curve), if $\theta$ is a constant function (see [5-7]).

Let $\gamma: I \rightarrow M$ be a slant curve on $M$ such that $g(\dot{\gamma}, \dot{\gamma})=\varepsilon_{1}= \pm 1$. Let us consider the vector fields $\dot{\gamma}, \varphi \dot{\gamma}, \xi$ for which, we have

$$
\begin{aligned}
g(\dot{\gamma}, \dot{\gamma}) & =\varepsilon_{1}, g(\dot{\gamma}, \xi)=c, g(\varphi \dot{\gamma}, \varphi \dot{\gamma})=-\varepsilon_{1}+c^{2}, g(\xi, \xi) \\
& =1, g(\dot{\gamma}, \varphi \dot{\gamma})=g(\xi, \varphi \dot{\gamma})=0,
\end{aligned}
$$

where $c$ is constant. These vector fields are linearly independent and form a bases of $T_{\gamma(t)} M$ for any $t \in I$ if and only if $\varepsilon_{1}-c^{2} \neq 0$. In this case, we define an orthonormal frame along $\gamma$ as follows:

$$
F_{1}=\dot{\gamma}, \quad F_{2}=\frac{\varphi \dot{\gamma}}{\sqrt{\left|\varepsilon_{1}-c^{2}\right|}}, \quad F_{3}=\frac{\xi-\varepsilon_{1} c \dot{\gamma}}{\sqrt{\left|\varepsilon_{1}-c^{2}\right|}},
$$


where $g\left(F_{1}, F_{1}\right)=\varepsilon_{1}, g\left(F_{2}, F_{2}\right)=\operatorname{sgn}\left(-\left(\varepsilon_{1}-c^{2}\right)\right)=v, g\left(F_{3}, F_{3}\right)=-\varepsilon_{1} v$.

The frame $\left(F_{1}, F_{2}, F_{3}\right)$ will play an auxiliary role in the proofs of Theorems 2.3 and 4.2 .

Separately, we discuss the case when $\dot{\gamma}, \varphi \dot{\gamma}, \xi$ are linearly dependent. Let us note that $\dot{\gamma}, \varphi \dot{\gamma}, \xi$ are linearly dependent if and only if

$$
\dot{\gamma}=c \xi \quad \text { or } \quad \dot{\gamma}=c \xi \pm \varphi \dot{\gamma} .
$$

Indeed, if $\dot{\gamma}, \varphi \dot{\gamma}, \xi$ are linearly dependent then $\varepsilon_{1}=1$ and $c^{2}=1$. Hence $g(\varphi \dot{\gamma}, \varphi \dot{\gamma})=0$ and either $\varphi \dot{\gamma}=0$ or $\varphi \dot{\gamma}$ is an isotropic vector field.

If $\varphi \dot{\gamma}=0$ then $0=\varphi^{2} \dot{\gamma}=\dot{\gamma}-c \xi$ and $\dot{\gamma}=c \xi$.

If $\varphi \dot{\gamma}$ is an isotropic vector field then, we can write $\dot{\gamma}=a \xi+b \varphi \dot{\gamma}$, for some functions $a$ and $b$. Using $g(\dot{\gamma}, \dot{\gamma})=1$ and $g(\dot{\gamma}, \xi)=c$, we find $a=c$. Now

$$
\varphi \dot{\gamma}=b \varphi^{2} \dot{\gamma}=b(\dot{\gamma}-c \xi)=b(c \xi+b \varphi \dot{\gamma}-c \xi)=b^{2} \varphi \dot{\gamma}
$$

Hence $b^{2}=1$ and $\dot{\gamma}=c \xi \pm \varphi \dot{\gamma}$.

\section{Frenet Slant Curves}

Let $(M, g)$ be a 3-dimensional pseudo-Riemannian manifold.

Let $\gamma: I \rightarrow M$ be a curve on $M, I$ being an interval.

We say that $\gamma$ is a Frenet curve if $g(\dot{\gamma}, \dot{\gamma})=\varepsilon_{1}, \varepsilon_{1}= \pm 1$ and one of the following three cases holds

(a) $\gamma$ is of osculating order 1, i.e., $\nabla_{\dot{\gamma}} \dot{\gamma}=0$ i.e. $\gamma$ is a geodesic;

(b) $\gamma$ is of osculating order 2, i.e., there exist two orthonormal vector fields $E_{1}, E_{2}$ and a positive function $\kappa$ (the curvature) along $\gamma$ such that $E_{1}=\dot{\gamma}, g\left(E_{2}, E_{2}\right)=\varepsilon_{2}= \pm 1$ and

$$
\nabla_{\dot{\gamma}} E_{1}=\kappa \varepsilon_{2} E_{2}, \quad \nabla_{\dot{\gamma}} E_{2}=-\kappa \varepsilon_{1} E_{1}
$$

(c) $\gamma$ is of osculating order 3, i.e., there exist three orthonormal vector fields $E_{1}, E_{2}, E_{3}$ and two positive functions $\kappa$ (the curvature) and $\tau$ (the torsion) along $\gamma$ such that $E_{1}=\dot{\gamma}, g\left(E_{2}, E_{2}\right)=\varepsilon_{2}= \pm 1, g\left(E_{3}, E_{3}\right)=\varepsilon_{3}= \pm 1$ and

$$
\nabla_{\dot{\gamma}} E_{1}=\kappa \varepsilon_{2} E_{2}, \quad \nabla_{\dot{\gamma}} E_{2}=-\kappa \varepsilon_{1} E_{1}+\tau \varepsilon_{3} E_{3}, \quad \nabla_{\dot{\gamma}} E_{3}=-\tau \varepsilon_{2} E_{2} .
$$

Proposition 2.1. Let $M$ be a 3-dimensional normal almost paracontact metric manifold. If $\gamma: I \rightarrow M$ is a slant Frenet curve in $M$, such that $g(\dot{\gamma}, \dot{\gamma})=1$ and $g(\dot{\gamma}, \xi)=c= \pm 1$ then $\gamma$ is a geodesics.

Proof In view of (1.6), we consider two cases.

If $\dot{\gamma}=c \xi$ then from (1.3) $\nabla_{\dot{\gamma}} \dot{\gamma}=\nabla_{\xi} \xi=0$ and $\gamma$ is a geodesics.

If $\dot{\gamma}=c \xi \pm \varphi \dot{\gamma}$ using (1.3) and (1.2), we get

$$
\begin{aligned}
\nabla_{\dot{\gamma}} \dot{\gamma} & =c \nabla_{\dot{\gamma}} \xi \pm \nabla_{\dot{\gamma}} \varphi \dot{\gamma}=c \nabla_{\dot{\gamma}} \xi \pm\left(\nabla_{\dot{\gamma}} \varphi\right) \dot{\gamma} \pm \varphi \nabla_{\dot{\gamma}} \dot{\gamma} \\
& =(\alpha \mp \beta)(c \dot{\gamma}-\xi \mp c \varphi \dot{\gamma}) \pm \varphi \nabla_{\dot{\gamma}} \dot{\gamma}= \pm \varphi \nabla_{\dot{\gamma}} \dot{\gamma} .
\end{aligned}
$$

Then $g\left(\nabla_{\dot{\gamma}} \dot{\gamma}, \nabla_{\dot{\gamma}} \dot{\gamma}\right)= \pm g\left(\nabla_{\dot{\gamma}} \dot{\gamma}, \varphi \nabla_{\dot{\gamma}} \dot{\gamma}\right)=0$ and taking the definition of Frenet curve into account, $\gamma$ is necessarily a geodesic. 
Proposition 2.2. Let $M$ be a 3-dimensional normal almost paracontact metric manifold. If $\gamma: I \rightarrow M$ is a slant Frenet curve of osculating order 3, then

$$
\begin{aligned}
& \nabla_{\dot{\gamma}} F_{1}=v \delta \sqrt{\left|\varepsilon_{1}-c^{2}\right|} F_{2}-\varepsilon_{1} \alpha \sqrt{\left|\varepsilon_{1}-c^{2}\right|} F_{3}, \\
& \nabla_{\dot{\gamma}} F_{2}=-\varepsilon_{1} \delta \sqrt{\left|\varepsilon_{1}-c^{2}\right|} F_{1}+\left(\varepsilon_{1} \beta-v c \delta\right) F_{3}, \\
& \nabla_{\dot{\gamma}} F_{3}=-\varepsilon_{1} v \alpha \sqrt{\left|\varepsilon_{1}-c^{2}\right|} F_{1}+\left(\beta-\varepsilon_{1} v c \delta\right) F_{2},
\end{aligned}
$$

where $\delta=\frac{g\left(\nabla_{\dot{\gamma}} \dot{\gamma}, \varphi \dot{\gamma}\right)}{\left|\varepsilon_{1}-c^{2}\right|}$, and for simplicity we write $\alpha, \beta$ instead of the composed functions $\alpha \circ \gamma, \beta \circ \gamma$ with $\alpha, \beta$ being the same as in (1.4).

Proof Let us put $\nabla_{\dot{\gamma}} F_{1}=p F_{1}+q F_{2}+r F_{3}$ for some functions $p, q, r$. Then $p \varepsilon_{1}=g\left(\nabla_{\dot{\gamma}} F_{1}, F_{1}\right)=0$.

Next using (1.2) we get

$$
\begin{aligned}
q v & =g\left(\nabla_{\dot{\gamma}} F_{1}, F_{2}\right)=-\frac{1}{\sqrt{\left|\varepsilon_{1}-c^{2}\right|}} g\left(\dot{\gamma}, \nabla_{\dot{\gamma}} \varphi \dot{\gamma}\right) \\
& =-\frac{1}{\sqrt{\left|\varepsilon_{1}-c^{2}\right|}} g\left(\dot{\gamma},\left(\nabla_{\dot{\gamma} \varphi} \varphi \dot{\gamma}+\varphi \nabla_{\dot{\gamma}} \dot{\gamma}\right)=\delta \sqrt{\left|\varepsilon_{1}-c^{2}\right|} .\right.
\end{aligned}
$$

Now using (1.3), we have

$$
\begin{aligned}
-\varepsilon_{1} v r & =g\left(\nabla_{\dot{\gamma}} F_{1}, F_{3}\right)=\frac{1}{\sqrt{\left|\varepsilon_{1}-c^{2}\right|}} g\left(\nabla_{\dot{\gamma}} \dot{\gamma}, \xi-\varepsilon_{1} c \dot{\gamma}\right) \\
& =-\frac{1}{\sqrt{\left|\varepsilon_{1}-c^{2}\right|}} g\left(\dot{\gamma}, \nabla_{\dot{\gamma}} \xi\right)=-\frac{1}{\sqrt{\left|\varepsilon_{1}-c^{2}\right|}} \alpha\left(\varepsilon_{1}-c^{2}\right) .
\end{aligned}
$$

Hence in view of above $p=0, q=v \delta \sqrt{\left|\varepsilon_{1}-c^{2}\right|}, r=-\varepsilon_{1} \alpha \sqrt{\left|\varepsilon_{1}-c^{2}\right|}$ and this leads to (2.1). Similarly, we calculate (2.1) and (2.3).

Theorem 2.3 generalizes results obtained for Legendre curves in [22].

Theorem 2.3. Let $M$ be a 3-dimensional normal almost paracontact metric manifold. If $\gamma: I \rightarrow M$ is a slant Frenet curve of osculating order 3 in $M$, then its curvature and torsion are given by

$$
\begin{aligned}
& \kappa=\sqrt{\left|\varepsilon_{1}-c^{2}\right|\left|\alpha^{2}-\varepsilon_{1} \delta^{2}\right|}, \\
& \tau=\left|\operatorname{sgn}\left(1-\varepsilon_{1} c^{2}\right) \beta+c \delta+\frac{\alpha \dot{\delta}-\dot{\alpha} \delta}{\alpha^{2}-\varepsilon_{1} \delta^{2}}\right| .
\end{aligned}
$$

where $\delta=\frac{g\left(\nabla_{\dot{\gamma}} \dot{\gamma}, \varphi \dot{\gamma}\right)}{\left|\varepsilon_{1}-c^{2}\right|}$, and for simplicity we write $\alpha, \beta$ instead of the composed functions $\alpha \circ \gamma, \beta \circ \gamma$ with $\alpha, \beta$ being the same as in (1.4).

Proof Let $\gamma$ be a slant Frenet curve of osculating order 3 in $M$. Then $\gamma$ is not a geodesic curve and from Proposition $2.1 \varepsilon_{1} \neq 1$ or $c \neq \pm 1$. Hence $\varepsilon_{1}-c^{2} \neq 0$ and so that we can use the frame $\left(F_{1}, F_{2}, F_{3}\right)$ from (1.5) to calculate the curvature and the torsion functions of $\gamma$.

We obtain the curvature function using (2.1) and calculating the length of $\nabla_{\dot{\gamma}} \dot{\gamma}$, we get

$$
\kappa^{2} \varepsilon_{2}=-\varepsilon_{1} v\left|\varepsilon_{1}-c^{2}\right|\left(\alpha^{2}-\varepsilon_{1} \delta^{2}\right)
$$


so that the curvature is given by (2.4) and $\varepsilon_{2}=\operatorname{sgn}\left(-\varepsilon_{1} v\left(\alpha^{2}-\varepsilon_{1} \delta^{2}\right)\right)= \pm 1$.

Using (2.1), (2.1) and (2.3), we find

$$
E_{2}=\frac{1}{\varepsilon_{2} \kappa} \nabla_{\dot{\gamma}} E_{1}=\frac{\varepsilon_{2}\left(v \delta F_{2}-\varepsilon_{1} \alpha F_{3}\right)}{\sqrt{\left|\alpha^{2}-\varepsilon_{1} \delta^{2}\right|}},
$$

Let us denote $p=\sqrt{\left|\alpha^{2}-\varepsilon_{1} \delta^{2}\right|}$ and calculate

$$
\begin{aligned}
\nabla_{\dot{\gamma}} E_{2}= & \varepsilon_{2} v \dot{\gamma} \frac{\delta}{p} F_{2}+\varepsilon_{2} v \frac{\delta}{p} \nabla_{\dot{\gamma}} F_{2} \\
& -\varepsilon_{1} \varepsilon_{2} \dot{\gamma} \frac{\alpha}{p} F_{3}-\varepsilon_{1} \varepsilon_{2} \frac{\alpha}{p} \nabla_{\dot{\gamma}} F_{3} \\
= & -\varepsilon_{1} \kappa E_{1}+\left(\varepsilon_{2} v \dot{\gamma} \frac{\delta}{p}-\varepsilon_{2} \frac{\alpha v}{p}\left(\varepsilon_{1} v \beta-c \delta\right)\right) F_{2} \\
& +\left(-\varepsilon_{1} \varepsilon_{2} \dot{\gamma} \frac{\alpha}{p}+\varepsilon_{2} \frac{\delta}{p}\left(\varepsilon_{1} v \beta-c \delta\right)\right) F_{3} \\
= & -\varepsilon_{1} \kappa E_{1}+\varepsilon_{2}\left(-\varepsilon_{1} v \beta+c \delta+\frac{\alpha \dot{\delta}-\dot{\alpha} \delta}{\alpha^{2}-\varepsilon_{1} \delta^{2}}\right) \frac{v \alpha F_{2}-\delta F_{3}}{\sqrt{\left|\alpha^{2}-\varepsilon_{1} \delta^{2}\right|}} .
\end{aligned}
$$

Hence from (2.6), in view of the Frenet equations, we have

$$
\begin{aligned}
\varepsilon_{3} \tau^{2} & =v \operatorname{sgn}\left(\alpha^{2}-\varepsilon_{1} \delta^{2}\right)\left(-\varepsilon_{1} v \beta+c \delta+\frac{\alpha \dot{\delta}-\dot{\alpha} \delta}{\alpha^{2}-\varepsilon_{1} \delta^{2}}\right)^{2} \\
& =-\varepsilon_{1} \varepsilon_{2}\left(\operatorname{sgn}\left(1-\varepsilon_{1} c^{2}\right) \beta+c \delta+\frac{\alpha \dot{\delta}-\dot{\alpha} \delta}{\alpha^{2}-\varepsilon_{1} \delta^{2}}\right)^{2} .
\end{aligned}
$$

This gives (2.5) and $\varepsilon_{3}=-\varepsilon_{1} \varepsilon_{2}$.

Below, we find the functions of curvature and torsion of a slant Frenet curve of osculating order 3 in some subclasses of a 3 -dimensional normal almost paracontact metric manifold.

Corollary 2.4. Let $M$ be a 3-dimensional manifold, $\gamma: I \rightarrow M$ be a slant Frenet curve of osculating order 3 in $M$.

If $M$ is a paracosymplectic manifold then the curvature and torsion of $\gamma$ are given by

$$
\kappa=\sqrt{\left|\varepsilon_{1}-c^{2}\right|}|\delta|, \quad \tau=|c \delta| .
$$

If $M$ is a quasi-para-Sasakian manifold then the curvature and torsion of $\gamma$ are given by

$$
\kappa=\sqrt{\left|\varepsilon_{1}-c^{2}\right|} \delta|, \quad \tau=| \operatorname{sgn}\left(1-\varepsilon_{1} c^{2}\right) \beta+c \delta \mid .
$$

If $M$ is a para-Sasakian manifold then the curvature and torsion of $\gamma$ are given by

$$
\kappa=\sqrt{\left|\varepsilon_{1}-c^{2}\right|}|\delta|, \quad \tau=\left|\operatorname{sgn}\left(1-\varepsilon_{1} c^{2}\right)+c \delta\right| .
$$


If $M$ is an $\alpha$-Kenmotsu manifold then the curvature and torsion of $\gamma$ are given by

$$
\kappa=\sqrt{\left|\varepsilon_{1}-c^{2}\right|\left|\alpha^{2}-\varepsilon_{1} \delta^{2}\right|}, \quad \tau=\left|c \delta+\frac{\alpha \dot{\delta}}{\alpha^{2}-\varepsilon_{1} \delta^{2}}\right|,
$$

where $\delta=\frac{g\left(\nabla_{\dot{\gamma}} \dot{\gamma}, \varphi \dot{\gamma}\right)}{\left|\varepsilon_{1}-c^{2}\right|}$ and for simplicity we write $\alpha, \beta$ instead of the composed functions $\alpha \circ \gamma, \beta \circ \gamma$ with $\alpha, \beta$ being the same as in (1.4).

\section{Null Slant Curves}

Next, we consider the case when $\gamma$ is a null curve, i.e has a null tangent vector field $(g(\dot{\gamma}, \dot{\gamma})=0)$ and $\gamma$ is not a geodesic, that is $g\left(\nabla_{\dot{\gamma}} \dot{\gamma}, \nabla_{\dot{\gamma}} \dot{\gamma}\right) \neq 0$.

We take a parametrization of $\gamma$ such that $g\left(\nabla_{\dot{\gamma}} \dot{\gamma}, \nabla_{\dot{\gamma}} \dot{\gamma}\right)=1$. Then in [13] it is proved that there exist only one Cartan frame $T, N, W$ and the function $\widetilde{\tau}$ along $\gamma$ such that following Cartan equations are satisfied

$$
\nabla_{\dot{\gamma}} T=N, \quad \nabla_{\dot{\gamma}} W=\widetilde{\tau} N . \quad \nabla_{\dot{\gamma}} N=-\widetilde{\tau} T-W .
$$

The above Cartan frame is defined as follows

$$
T=\dot{\gamma}, \quad N=\nabla_{\dot{\gamma}} T, \quad \widetilde{\tau}=\frac{1}{2} g\left(\nabla_{\dot{\gamma}} N, \nabla_{\dot{\gamma}} N\right), \quad W=-\nabla_{\dot{\gamma}} N-\widetilde{\tau} T .
$$

Hence

$$
g(T, W)=g(N, N)=1, \quad g(T, T)=g(T, N)=g(W, W)=g(W, N)=0 .
$$

The Cartan frame is a frame with the minimum number of curvature functions which are invariant under Lorentzian transformation (see [11-13, 19]).

In [22], we proved that every null Legendre curve in a 3-dimensional normal almost paracontact metric manifold is a geodesic.

In this section, we consider a slant non-geodesic null curve $\gamma: I \rightarrow M$.

Theorem 3.1. If $\gamma: I \rightarrow M$ is a slant non-geodesic null curve on a 3dimensional normal almost paracontact metric manifold then

$$
\begin{aligned}
\widetilde{\tau} & =-\frac{\alpha^{2} c^{2}}{2}-\dot{\alpha} c \pm \beta-\frac{1}{2 c^{2}}, \\
N & =\alpha c \dot{\gamma} \pm \frac{1}{c} \varphi \dot{\gamma}, \\
W & =\frac{-\alpha^{2} c^{2}-1}{2 c} \dot{\gamma} \mp \alpha \varphi \dot{\gamma}+\frac{1}{c^{2}} \xi,
\end{aligned}
$$

where for simplicity we write $\alpha, \beta$ instead of the composed functions $\alpha \circ \gamma$, $\beta \circ \gamma$ with $\alpha, \beta$ being the same as in (1.4).

Proof Let $\varphi \dot{\gamma}=p \dot{\gamma}+q N+r W$ for some functions $p, q, r$. We find $r=$ $g(\varphi \dot{\gamma}, \dot{\gamma})=0$. Then $\varphi \dot{\gamma}=p \dot{\gamma}+q N$ and

$$
g(\varphi \dot{\gamma}, \varphi \dot{\gamma})=c^{2}=q^{2}, \quad 0=g(\varphi \dot{\gamma}, \xi)=p c+q \alpha c^{2}=c\left(p \pm \alpha c^{2}\right) .
$$

Hence $q= \pm c, p=\mp \alpha c^{2}$ and eventually $\varphi \dot{\gamma}=\mp \alpha c^{2} \dot{\gamma} \pm c N$, which lead to $(3.3)$. 
Now, applying (1.2) and (3.3), we calculate

$$
\begin{aligned}
\nabla_{\dot{\gamma}} N & =\dot{\alpha} c \dot{\gamma}+\alpha c \nabla_{\dot{\gamma}} \dot{\gamma} \pm \frac{1}{c}\left(\left(\nabla_{\dot{\gamma}} \varphi\right) \dot{\gamma}+\varphi \nabla_{\dot{\gamma}} \dot{\gamma}\right) \\
& =\left(\dot{\alpha} c a+\alpha^{2} c^{2} \mp \beta+\frac{1}{c}\right) \dot{\gamma} \pm \alpha \varphi \dot{\gamma}-\frac{1}{c} \xi .
\end{aligned}
$$

Next, in view of the definition $\widetilde{\tau}$ and $W$ in (3.1), we use (3.5) and get (3.2) and (3.4).

In the following corollary, we present the properties of slant null curves in some subclasses of a 3-dimensional normal almost paracontact metric manifold.

Corollary 3.2. Let $M$ be a 3-dimensional manifold, $\gamma: I \rightarrow M$ be a slant non-geodesic null curve on $M$.

If $M$ is a paracosymplectic manifold then for $\gamma$, we have

$$
\widetilde{\tau}=-\frac{1}{2 c^{2}}, \quad N= \pm \frac{1}{c} \varphi \dot{\gamma}, \quad W=\frac{1}{c^{2}} \xi .
$$

If $M$ is a quasi-para-Sasakian manifold then for $\gamma$, we have

$$
\widetilde{\tau}= \pm \beta-\frac{1}{2 c^{2}}, \quad N= \pm \frac{1}{c} \varphi \dot{\gamma}, \quad W=\frac{1}{c^{2}} \xi .
$$

If $M$ is a para-Sasakian manifold then for $\gamma$, we have

$$
\widetilde{\tau}= \pm 1-\frac{1}{2 c^{2}}, \quad N= \pm \frac{1}{c} \varphi \dot{\gamma}, \quad W=\frac{1}{c^{2}} \xi .
$$

If $M$ is an $\alpha$-Kenmotsu manifold then for $\gamma$, we have

$$
\widetilde{\tau}=-\frac{\alpha^{2} c^{2}}{2}-\frac{1}{2 c^{2}}, \quad N=\alpha c \dot{\gamma} \pm \frac{1}{c} \varphi \dot{\gamma}, \quad W=\frac{-\alpha^{2} c^{2}-1}{2 c} \dot{\gamma} \mp \alpha \varphi \dot{\gamma}+\frac{1}{c^{2}} \xi .
$$

\section{Slant Curves with Null Normal}

At last, we investigate curves with null normals (for such curves on Minkowski space $\mathbb{E}_{1}^{3}$, see among others $\left.[3,17,18]\right)$. We say that $\gamma: I \rightarrow M$ is a curve with null normal if

$$
g(\dot{\gamma}, \dot{\gamma})=1, \quad \nabla_{\dot{\gamma}} \dot{\gamma} \neq 0, \quad g\left(\nabla_{\dot{\gamma}} \dot{\gamma}, \nabla_{\dot{\gamma}} \dot{\gamma}\right)=0 .
$$

Proposition 4.1. Let $\gamma: I \rightarrow M$ be a curve with null normal. Then there exist the frame $T, N, V$ and the curvature function $\widetilde{\kappa}$ along $\gamma$, which satisfy the following Cartan equations:

$$
\nabla_{\dot{\gamma}} T=N, \quad \nabla_{\dot{\gamma}} N=\widetilde{\kappa} N, \quad \nabla_{\dot{\gamma}} V=-T-\widetilde{\kappa} V .
$$

Proof By the assumption $\nabla_{\dot{\gamma}} \dot{\gamma}$ is a null vector field. We set $N=\nabla_{\dot{\gamma}} \dot{\gamma}$. Since $T=\dot{\gamma}$ is a unit vector field, there exist a unique null vector field $V$ along $\gamma$ (see [16]) such that

$$
g(N, V)=1 \quad \text { and } \quad g(T, V)=0 .
$$


Next we check that $T, N, V$ satisfies (4.1). We put $\nabla_{\dot{\gamma}} N=a T+b N+c V$ for some functions $a, b, c$. Then

$$
a=g\left(\nabla_{\dot{\gamma}} N, T\right)=-g(N, N)=0, \quad c=g\left(\nabla_{\dot{\gamma}} N, N\right)=0 .
$$

Hence $\nabla_{\dot{\gamma}} N=b N$. Similarly, we calculate $\nabla_{\dot{\gamma}} V=-T-b V$. Thus $T, N, V$ satisfies (4.1) with $\widetilde{\kappa}=b$.

Theorem 4.2. If $\gamma: I \rightarrow M$ is a slant non-geodesic curve with null normal in a 3-dimensional normal almost paracontact metric manifold, for which $c^{2} \neq 1$ then

$$
\begin{aligned}
N & =-\alpha(\xi-c \dot{\gamma} \pm \varphi \dot{\gamma}), \\
\widetilde{\kappa} & =\frac{\dot{\alpha}}{\alpha} \pm \beta+\alpha c, \\
V & =\frac{-1}{2 \alpha\left(1-c^{2}\right)}(\xi-c \dot{\gamma} \mp \varphi \dot{\gamma}),
\end{aligned}
$$

where for simplicity we write $\alpha, \beta$ instead of the composed functions $\alpha \circ \gamma$, $\beta \circ \gamma$ with $\alpha, \beta$ being the same as in (1.4). Moreover $g(N, \varphi \dot{\gamma})= \pm \alpha\left(1-c^{2}\right) \neq$ 0 .

Proof For a slant curve with null normal for which $c^{2} \neq 1$, we can use an orthonormal frame along $\gamma$ as in (1.5). We put

$$
N=\nabla_{\dot{\gamma}} \dot{\gamma}=a F_{2}+b F_{3},
$$

for some functions $a$ and $b$ and calculate $g(N, N)=a^{2}-b^{2}=0$. Since $\gamma$ is not a geodesic then $a= \pm b \neq 0$. Moreover, using (1.3), we get

$$
\begin{aligned}
0 \neq b=g\left(N, F_{3}\right) & =-\frac{1}{\sqrt{\left|1-c^{2}\right|}} g\left(\nabla_{\dot{\gamma}} \xi, \dot{\gamma}\right) \\
& =-\alpha \sqrt{\left|1-c^{2}\right|} .
\end{aligned}
$$

Hence $\alpha \neq 0$ and putting (4.7) in (4.6), we obtain (4.3).

Next, using (1.5), we have

$$
a v=g\left(N, F_{2}\right)=\frac{g(N, \varphi \dot{\gamma})}{\sqrt{\left|1-c^{2}\right|}}
$$

and putting (4.7) in (4.8), we get $g(N \varphi \dot{\gamma})= \pm \alpha\left(1-c^{2}\right) \neq 0$.

To find the curvature expression of the $\widetilde{\kappa}$, we use (1.3), (4.3), (1.2) and calculate

$$
\begin{aligned}
\nabla_{\dot{\gamma}} N= & -\dot{\alpha}(\xi-c \dot{\gamma} \pm \varphi \dot{\gamma}) \\
& -\alpha\left(\nabla_{\dot{\gamma}} \xi-c \nabla_{\dot{\gamma}} \dot{\gamma} \pm\left(\nabla_{\dot{\gamma}} \varphi\right) \dot{\gamma} \pm \varphi \nabla_{\dot{\gamma}} \dot{\gamma}\right) \\
= & \left(-\dot{\alpha} \mp \alpha \beta-\alpha^{2} c\right)(\xi-c \dot{\gamma} \pm \varphi \dot{\gamma}) \\
= & \left(\frac{\dot{\alpha}}{\alpha} \pm \beta+\alpha c\right) N .
\end{aligned}
$$

In view of (4.1), we obtain (4.4).

At last, we put $V=d F_{1}+e F_{2}+f F_{3}$ for some functions $d, e, f$ and using (4.2), we get (4.5).

As an immediate consequence of the above theorem, we obtain 
Theorem 4.3. Let $\gamma: I \rightarrow M$ be a Legendre curve with null normal in a 3-dimensional normal almost paracontact metric manifold $M$. Then $\alpha=$ $\pm g(N, \varphi \dot{\gamma}), \alpha \neq 0$ and we have

$$
T=\dot{\gamma}, \quad N=-\alpha(\xi \pm \varphi \dot{\gamma}), \quad V=\frac{-1}{2 \alpha}(\xi \mp \varphi \dot{\gamma}) \quad \text { and } \quad \widetilde{\kappa}=\frac{\dot{\alpha}}{\alpha} \pm \beta,
$$

where for simplicity we write $\alpha, \beta$ instead of the composed functions $\alpha \circ \gamma$, $\beta \circ \gamma$ with $\alpha, \beta$ being the same as in (1.4).

Corollary 4.4. Let $\gamma: I \rightarrow M$ be a slant curve with null normal in a 3dimensional $\alpha$-para-Kenmotsu manifold $M$, for which $c^{2} \neq 1$. Then $g(N, \varphi \dot{\gamma})$ $= \pm \alpha\left(1-c^{2}\right) \in \mathbb{R} \backslash\{0\}$

$$
\widetilde{\kappa}=\alpha c, \quad N=-\alpha(\xi-c \dot{\gamma} \pm \varphi \dot{\gamma}), \quad V=\frac{-1}{2 \alpha\left(1-c^{2}\right)}(\xi-c \dot{\gamma} \mp \varphi \dot{\gamma}) .
$$

In the particular case when $\gamma$ is a Legendre curve, we have

$$
\widetilde{\kappa}=0, \quad N=-\alpha(\xi \pm \varphi \dot{\gamma}), \quad V=\frac{-1}{2 \alpha}(\xi \mp \varphi \dot{\gamma}) .
$$

where for simplicity we write $\alpha, \beta$ instead of the composed functions $\alpha \circ \gamma$, $\beta \circ \gamma$ with $\alpha, \beta$ being the same as in (1.4).

\section{Examples}

Legendre curves with null normal that will appear in examples are from my previous paper [22].

Example 1. Let $\mathbb{R}^{3}$ be the Cartesian space with the standard Cartesian coordinates $(x, y, z)$. Define the standard almost paracontact structure $(\varphi, \xi, \eta)$ on $\mathbb{R}^{3}$ by

$$
\varphi \partial_{1}=\partial_{2}-2 x \partial_{3}, \quad \varphi \partial_{2}=\partial_{1}, \quad \varphi \partial_{3}=0, \quad \xi=\partial_{3}, \quad \eta=2 x d y+d z,
$$

where $\partial_{1}=\frac{\partial}{\partial x}, \partial_{2}=\frac{\partial}{\partial y}$ and $\partial_{3}=\frac{\partial}{\partial z}$. By straightforward calculations, one verifies that

$$
[\varphi, \varphi]\left(\partial_{i}, \partial_{j}\right)-2 d \eta\left(\partial_{i}, \partial_{j}\right) \xi=0, \quad 1 \leqslant i<j \leqslant 3,
$$

so that (1.1) is satisfied and the structure is normal.

Let $M=\mathbb{R}^{2} \times \mathbb{R}_{-} \subset \mathbb{R}^{3}$ and consider a normal almost paracontact metric structure on $M$ defined in the following way: $(\varphi, \xi, \eta)$ is the structure (5.1) restricted to $M$ and $g$ is the Lorentz metric given by

$$
\left[g\left(\partial_{i}, \partial_{j}\right)\right]=\left[\begin{array}{lll}
-2 z & 0 & 0 \\
0 & 4 x^{2}+2 z & 2 x \\
0 & 2 x & 1
\end{array}\right] .
$$


For the Levi-Civita connection, we have

$$
\begin{gathered}
\nabla_{\partial_{1}} \partial_{1}=-\frac{x}{z} \partial_{2}+\left(1+\frac{2 x^{2}}{z}\right) \partial_{3}, \quad \nabla_{\partial_{1}} \partial_{2}=\nabla_{\partial_{2}} \partial_{1}=\frac{x}{z} \partial_{2}+\left(1-\frac{2 x^{2}}{z}\right) \partial_{3}, \\
\nabla_{\partial_{1}} \partial_{3}=\nabla_{\partial_{3}} \partial_{1}=\nabla_{\partial_{2}} \partial_{3}=\nabla_{\partial_{3}} \partial_{2}=\frac{1}{2 z} \partial_{1}+\frac{1}{2 z} \partial_{2}-\frac{x}{z} \partial_{3}, \\
\nabla_{\partial_{2}} \partial_{2}=\frac{2 x}{z} \partial_{1}+\frac{x}{z} \partial_{2}-\left(1+\frac{2 x^{2}}{z}\right) \partial_{3}, \quad \nabla_{\partial_{3}} \partial_{3}=0 .
\end{gathered}
$$

Using the above expressions and (1.3), we find $\alpha=\beta=(2 z)^{-1}$.

(a) $\gamma(t)=(0,-2 \sqrt{-t}, t), \quad t<0$

is a Frenet slant curve of osculating order 3. For such a curve $\varepsilon_{1}=-1$, $c=1, \alpha(\gamma(t))=\beta(\gamma(t))=\frac{1}{2 t}, \delta(t)=\frac{1}{t}, \kappa=-\frac{\sqrt{5}}{\sqrt{2} t}, \tau=-\frac{3}{2 t}$.

Now let $M=\mathbb{R}^{2} \times \mathbb{R}_{+} \subset \mathbb{R}^{3}$ and consider a normal almost paracontact metric structure on $M$ such as in example $(a)$

We consider the following curves:

(b) $\gamma(t)=(1 / 4, t, 3 / 8)$,

is a slant curve with null normal and $\varepsilon_{1}=1$.

For such a curve, we have $c=1 / 2, \alpha(\gamma(t))=4 / 3, \beta(\gamma(t))=4 / 3$ and

$$
\begin{aligned}
& T=(0,1,0), \quad N=(4 / 3,2 / 3,-4 / 3) \\
& V=(-1 / 2,1 / 4,-1 / 2), \quad \widetilde{\kappa}=-2 / 3 .
\end{aligned}
$$

(c) $\gamma(t)=(\sqrt{t},-a \sqrt{t}, a t), \quad t>0, \quad a=\sqrt[3]{1-b}+\sqrt[3]{1+b}, \quad b=\sqrt{\frac{26}{27}}$,

is a Legendre curve with null normal and $\varepsilon_{1}=1$. For such a curve, we have $\alpha(\gamma(t))=(2 a t)^{-1}, \beta(\gamma(t))=(2 a t)^{-1}, \alpha(\gamma(t))=g(N, \varphi \dot{\gamma})=1 /(2 a t)$ and

$$
\begin{aligned}
& T=\left((2 \sqrt{t})^{-1},-a(2 \sqrt{t})^{-1}, a\right), \quad N=\left(1 / 4 t^{-3 / 2},-1 /(4 a) t^{-3 / 2}, 0\right), \\
& V=\left(-2 a^{2} \sqrt{t}, 2 a \sqrt{t},-8 a t\right), \quad \widetilde{\kappa}=\frac{1-2 a}{2 a t} .
\end{aligned}
$$

Example 2. Let $M=\mathbb{R}_{-} \times \mathbb{R}^{2}$. Define a normal almost paracontact structure $(\varphi, \xi, \eta)$ on $M$ by

$$
\varphi \partial_{1}=\partial_{2}, \quad \varphi \partial_{2}=\partial_{1}, \quad \varphi \partial_{3}=0, \quad \xi=\partial_{3}, \quad \eta=d z
$$

Let us define a compatible Lorentz metric

$$
\left[g\left(\partial_{i}, \partial_{j}\right)\right]=\left[\begin{array}{lll}
-x & 0 & 0 \\
0 & x & 0 \\
0 & 0 & 1
\end{array}\right]
$$

The quadruple $(\varphi, \xi, \eta, g)$ becomes a normal almost paracontact metric structure on $M$. For the Levi-Civita connection, we find

$$
\begin{aligned}
& \nabla_{\partial_{1}} \partial_{1}=\frac{1}{2 x} \partial_{1}, \quad \nabla_{\partial_{2}} \partial_{2}=\frac{1}{2 x} \partial_{1}, \quad \nabla_{\partial_{1}} \partial_{2}=\nabla_{\partial_{2}} \partial_{1}=\frac{1}{2 x} \partial_{2}, \\
& \nabla_{\partial_{1}} \partial_{3}=\nabla_{\partial_{3}} \partial_{1}=\nabla_{\partial_{2}} \partial_{3}=\nabla_{\partial_{3}} \partial_{2}=\nabla_{\partial_{3}} \partial_{3}=0
\end{aligned}
$$

Using the above and (1.3), we get $\alpha=\beta=0$. 
The curve

$$
\gamma(t)=\left(-\frac{1}{\sqrt[3]{4}},-\sqrt[3]{2} t, t\right)
$$

is a null slant curve on $M$. For such a curve $c=1$ and

$$
\begin{aligned}
& T=(0,-\sqrt[3]{2}, 1), \quad N=(-\sqrt[3]{2}, 0,0), \quad g(N, N)=1, \\
& W=(0,-\sqrt[3]{2} / 2,1 / 2), \quad \widetilde{\tau}=-1 / 2 .
\end{aligned}
$$

Example 3. Suppose that $M=\mathbb{R}^{2} \times \mathbb{R}_{+}$. Define a normal almost paracontact structure $(\varphi, \xi, \eta)$ on $M$ by

$$
\varphi \partial_{1}=\partial_{2}, \quad \varphi \partial_{2}=\partial_{1}, \quad \varphi \partial_{3}=0, \quad \xi=\partial_{3}, \quad \eta=d z,
$$

and compatible with this structure a Lorentz metric

$$
\left[g\left(\partial_{i}, \partial_{j}\right)\right]=\left[\begin{array}{ccc}
-2 z & 0 & 0 \\
0 & 2 z & 0 \\
0 & 0 & 1
\end{array}\right] .
$$

The quadruple $(\varphi, \xi, \eta, g)$ becomes a normal almost paracontact metric structure on $M$. For the Levi-Civita connection, we find

$$
\begin{aligned}
& \nabla_{\partial_{1}} \partial_{1}=\partial_{3}, \quad \nabla_{\partial_{1}} \partial_{2}=\nabla_{\partial_{2}} \partial_{1}=0, \quad \nabla_{\partial_{1}} \partial_{3}=\nabla_{\partial_{3}} \partial_{1}=\frac{1}{2 z} \partial_{1}, \\
& \nabla_{\partial_{2}} \partial_{2}=-\partial_{3}, \quad \nabla_{\partial_{2}} \partial_{3}=\nabla_{\partial_{3}} \partial_{2}=\frac{1}{2 z} \partial_{2}, \quad \nabla_{\partial_{3}} \partial_{3}=0 .
\end{aligned}
$$

Using the above and (1.3), we get $\alpha=(2 z)^{-1}$ and $\beta=0$.

The curve

$$
\gamma(t)=\left(\cosh t, \sinh t, \frac{1}{2}\right),
$$

is a Legendre curve with $\varepsilon_{1}=1$ and null normal in $M$.

Then for $\gamma$, we get $\alpha(\gamma(t))=1, \delta(t)=-1$ and

$$
\begin{aligned}
& T=(\sinh t, \cosh t, 0), \quad N=(\cosh t, \sinh t,-1), \\
& V=-\frac{1}{2}(\cosh t, \sinh t, 1), \quad \widetilde{\kappa}=0 .
\end{aligned}
$$

Open Access. This article is distributed under the terms of the Creative Commons Attribution License which permits any use, distribution, and reproduction in any medium, provided the original author(s) and the source are credited.

\section{References}

[1] Belkhelfa, M., Hirică, I.E., Rosa, R., Verstraelen, L.: On Legendre curves in Riemannian and Lorentzian Sasaki spaces. Soochow J. Math. 28(1), 81-91 (2002)

[2] Blair, D.E.: Riemannian Geometry of Contact and Symplectic Manifolds, vol. 203. Springer, Berlin (2010)

[3] Bonnor, W.B.: Curves with null normals in Minkowski space-time. In: Vaidya, E.H.P.C., RaychaudhuriA, A.K.(eds.) Random walk in relativity and cosmology. Wiley Easten Limited, New Delhi, pp. 33-47 (1985) 
[4] Cappelletti Montano, B.: Bi-Legendrian structures and paracontact geometry. Int. J. Geom. Meth. Mod. Phys. 6, 487-504 (2009)

[5] Călin, C., Crasmareanu, M.: Slant Curves in 3-dimensional Normal Almost Contact Geometry. Mediterr. J. Math. 10(2), 1067-1077 (2013)

[6] Călin, C., Crasmareanu, M.: Slant curves and particles in 3-dimensional warped products and their Lancret invariants. Bull. Austr. Math. Soc. Available on CJO2012. (2004). doi:10.1017/S0004972712000809

[7] Călin, C., Crasmareanu, M., Munteanu, M.: Slant curves in 3-dimensional f -Kenmotsu manifolds. J. Math. Anal. Appl. 394, 400-407 (2012)

[8] Cho, J.T., Inoguchi, J.I., Lee, J.E.: On slant curves in Sasakian space forms. J. Korean Math. Soc. 74(3), 359-367 (2006)

[9] Cho, J.T., Lee, J.E.: Slant curves in contact pseudo-Hermitian 3manifolds. Bull. Aust. Math. Soc. 78(3), 383-396 (2008)

[10] Dacko, P.: On almost para-cosymplectic manifolds. Tsukuba J. Math. 28(1):193-213 (2004)

[11] Duggal, K.L.: A Report on Canonical Null Curves and Screen Distributions for Lightlike Geometry. Acta Appl. Math. 95:135-149 (2007)

[12] Duggal, K.L., Bejancu, A.: Lightlike submanifolds of semi-Riemannian manifolds and applications. Mathematics and its Applications, vol. 364. Kluwer Academic Publishers Group, Dordrecht (1996)

[13] Duggal, K.L., Jin, D.H.: Null curves and hypersurfaces of semi-Riemannian manifolds. World Scientific Publishing, Singapore (2007)

[14] Erdem, S.: On almost (para)contact (hyperbolic) metric manifolds and harmonicity of $\left(\varphi, \varphi^{\prime}\right)$-holomorphic maps between them. Houston J. Math. 28, 2145 (2002)

[15] Fetcu, D.: Biharmonic Legendre curves in sasakian space forms. J. Korean Math. Soc. 45(2), 393-404 (2008)

[16] Graves, L.K.: Codimension one isometric immersions between Lorentz spaces. Trans. Am. Math.Soc. 252, 367-392 (1979)

[17] Ilarslan, K., Nešović, E.: Timelike and null normal curves in Minkowski space $\mathbb{E}_{1}^{3}$. Indian J. Pure Appl. Math. 35(7), 881-888 (2004)

[18] Inoguchi, J.I.: Biharmonic curves in Minkowski 3-space. II. Int. J. Math. Math. Sci. 2006, 1-4 (2006)

[19] Kocayigit, H., Hacisalihoglu, H.H.: 1-type and biharmonic frenet curves in Lorentzian 3-space. Iranian J. Sci. Technol. Trans. A Sci. 33, 159-168 (2009)

[20] Kaneyuki, S., Williams, F.L.: Almost paracontact and paraHodge structures on manifolds. Nagoya Math. J. 99, 173-187 (1985)

[21] Lee, H.: On Legendre curves in contact pseudo-Hermitian 3-manifolds. Bull. Aust. Math. Soc. 81(1):156-164 (2010)

[22] Wełyczko, J.: On Legendre curves in 3-dimensional normal almost paracontact metric manifolds. Results Math. 54, 377-387 (2009)

[23] Zamkovoy, S.: Canonical connections on paracontact manifolds. Ann. Glob. Anal. Geom. 36(1), 37-60 (2009) 


\section{Joanna Wełyczko}

Institute of Mathematics and Computer Science

Wrocław University of Technology

Wybrzeże Wyspiańskiego 27

50-370 Wrocław

Poland

e-mail: joanna.welyczko@pwr.wroc.pl

Received: March 9, 2013.

Revised: October 21, 2013.

Accepted: October 22, 2013. 